\title{
Amorphization and decomposition of scandium molybdate at high pressure
}

\author{
Akhilesh K. Arora ${ }^{a}$ \\ Materials Science Division, Indira Gandhi Centre for Atomic Research, Kalpakkam 603 102, India \\ Takehiko Yagi and Nobuyoshi Miyajima \\ Institute for Solid State Physics, Tokyo University, Kashowanoha, Kashiwa, Chiba 277-8581, Japan \\ T. A. Mary \\ Department of Materials Sciences, California Institute of Technology, Pasadena, California 91125
}

(Received 29 March 2004; accepted 23 September 2004; published online 13 December 2004)

\begin{abstract}
The behavior of negative thermal-expansion material scandium molybdate $\mathrm{Sc}_{2}\left(\mathrm{MoO}_{4}\right)_{3}$ is investigated at high pressure (HP) and high temperature (HT) using x-ray diffraction, Raman spectroscopy, and scanning electron microscopy. The compound exhibits unusually high compressibility (bulk modulus $\sim 6 \mathrm{GPa}$ ) and undergoes amorphization at $12 \mathrm{GPa}$. On the other hand, in situ laser heating of amorphous samples inside the diamond-anvil cell is found to result in crystalline diffraction pattern and Raman spectrum different from those of the original compound. Upon release of the pressure subsequent to laser heating, the Raman spectrum and the diffraction pattern remain unchanged. Matching of several of the diffraction lines and Raman peaks in the laser-heated samples with those of $\mathrm{MoO}_{3}$ suggests a solid-state decomposition of the parent compound under HP-HT conditions into $\mathrm{MoO}_{3}$ and other compounds. Other diffraction lines are found to correspond to $\mathrm{Sc}_{2} \mathrm{Mo}_{2} \mathrm{O}_{9}, \mathrm{Sc}_{2} \mathrm{O}_{3}$, and the parent compound. Quantitative analysis of the characteristic $\mathrm{x}$-ray emission from different regions of the sample during scanning electron microscopic observations is used for obtaining the compositions of the daughter compounds. The stoichiometries of two main phases are found to be close to those of $\mathrm{MoO}_{3}$ and $\mathrm{Sc}_{2} \mathrm{Mo}_{2} \mathrm{O}_{9}$. These results support the model that the pressure-induced amorphization occurred in this system because a pressure-induced decomposition was kinetically constrained. (C) 2005 American Institute of Physics. [DOI: 10.1063/1.1819975]
\end{abstract}

\section{INTRODUCTION}

Although the phenomenon of pressure-induced amorphization (PIA) is known to occur in nearly a hundred compounds, a complete understanding of its mechanisms and causes is yet to emerge. ${ }^{1,2}$ A wide variety of compounds with different types of bonding such as covalent $\left(\mathrm{SiO}_{2}\right)$, ionic $\left(\mathrm{LiKSO}_{4}\right)$, molecular $\left(\mathrm{SnI}_{4}\right)$, and hydrogen bonding $\left(\mathrm{H}_{2} \mathrm{O}\right)$ have exhibited the phenomenon. ${ }^{3-6}$ In addition, systems with more complex bonding such as those in silicates, tungstates, ${ }^{8}$ molybdates, ${ }^{9}$ and other minerals ${ }^{10}$ are also found to undergo amorphization at high pressure. A number of factors such as metastable melting, ${ }^{6}$ kinetic hindrance of phase transition, ${ }^{11}$ dynamical instability, ${ }^{12}$ polytetrahedral packing, ${ }^{13}$ and orientational disorder of polyatomic ions ${ }^{14}$ have been argued to be responsible for this. These features are not necessarily all distinct, i.e., more than one may be applicable simultaneously in some cases and hence could be related. Although kinetic hindrance of equilibrium phase transition ${ }^{11}$ has been frequently stated as the cause of PIA, the "final phase," which the system should have ideally evolved to, remains speculative or unknown in most instances. Recently, kinetic hindrance of decomposition/ disproportionation $^{15}$ of the parent compound into a mixture of dense-packed daughter compounds has been proposed to be the cause of PIA at ambient temperature in many com-

${ }^{\text {a)Electronic mail: aka@igcar.ernet.in }}$ pounds. As the process of nucleation and growth of macroscopic daughter phases is diffusion controlled, pressureinduced solid-state decomposition can only occur at elevated temperature. On the other hand, at ambient temperature, the system is likely to get trapped in a metastable amorphous (disordered) state while attempting to decompose under the application of pressure. The reported PIA in zirconium tungstate at $2.1 \mathrm{GPa}$ and ambient temperature ${ }^{16}$ and pressureinduced decomposition (PID) into mixture of simple oxides at $0.6 \mathrm{GPa}$ and $1073 \mathrm{~K}^{17}$ are consistent with this model. The condition $\Delta V<0$, where $\Delta V$ is the volume change upon decomposition, has been identified as a criterion for PIA at ambient temperature. ${ }^{1}$

Scandium molybdate is a negative thermal-expansion material that belongs to the family of compounds with network structure. It consists of corner-linked $\mathrm{ScO}_{6}$ octahedra and $\mathrm{MoO}_{4}$ tetrahedra. ${ }^{18}$ Several other network structures such as zirconium tungstate, ${ }^{16}$ scandium tungstate, ${ }^{19}$ and $\mathrm{Sm}$, $\mathrm{Eu}$, and $\mathrm{Gd}$ molybdates ${ }^{20}$ are found to undergo PIA at rather moderate pressures of 2-5 GPa. Among these scandium molybdate is a unique system which exhibits amorphization in two stages. ${ }^{21}$ The pressure-induced amorphization in these network structures has been argued to arise due to kinetic hindrance of decomposition. ${ }^{20,22}$ All these compounds are also found to satisfy the condition $\Delta V<0$ for amorphization. In view of this, it is of interest to examine whether scandium molybdate (a) undergoes PIA at ambient temperature and (b) exhibits decomposition under high-pressure high- 
temperature conditions. In analogy with the case of $\mathrm{Zr}\left(\mathrm{WO}_{4}\right)_{2}$, if one considers the decomposition of the parent compound into a mixture of simple oxides as

$$
\mathrm{Sc}_{2}\left(\mathrm{MoO}_{4}\right)_{3} \rightarrow \mathrm{Sc}_{2} \mathrm{O}_{3}+3 \mathrm{MoO}_{3},
$$

then the volume change upon decomposition can be calculated from volumes per formula unit of these compounds using their unit-cell data. Volumes per formula unit for $\mathrm{Sc}_{2}\left(\mathrm{MoO}_{4}\right)_{3}, \mathrm{Sc}_{2} \mathrm{O}_{3}$, and $\mathrm{MoO}_{3}$ are 304.96, 59.64, and $50.75 \AA^{3}$, respectively. From these, $\Delta V$ turns out to be $-30.5 \%$ of the parent volume, predicting amorphization/ decomposition under appropriate conditions. Although in the case of zirconium tungstate the decomposition products were simple oxides, in other systems it is possible that more complex-mixed oxides may emerge as daughter compounds. This can be sorted out only by carrying out actual highpressure high-temperature (HP-HT) experiment. In this paper we report detailed in situ high-pressure investigations on scandium molybdate using x-ray diffraction (XRD) and Raman spectroscopy in a diamond-anvil cell. The changes taking place upon laser heating the sample at high pressure are examined also in the recovered samples using scanning electron microscopy (SEM) in addition to $\mathrm{x}$-ray diffraction and Raman spectroscopy. Characteristic $\mathrm{x}$-ray emission is used for estimating the composition of phases formed due to HP-HT treatment. The results are discussed in the light of a recent model of amorphization/decomposition. ${ }^{15}$

\section{EXPERIMENTAL DETAILS}

Scandium molybdate was synthesized from the constituent oxides using standard solid-state technique described elsewhere. ${ }^{18}$ Powdered sample was loaded in a $300-\mu \mathrm{m}$ hole of preindented stainless-steel gasket of the diamond-anvil cell. A 4:1 methanol:ethanol mixture was used as the pressure-transmitting medium. Ruby fluorescence was used for the estimation of pressure. Raman-scattering measurements were carried out using a Photon-Design micro-Raman spectrometer that uses HR-320 monochromator and a liquidnitrogen-cooled charge-coupled device (CCD) detector. About $15 \mathrm{~mW}$ of power at $514.5-\mathrm{nm}$ wavelength from an argon-ion laser was used to excite the sample. X-ray diffraction measurements were made using Mo- $K_{\alpha}$ radiation from a 5.4-kW Rigaku rotating anode generator equipped with a $100-\mu \mathrm{m}$ collimator. An image plate (IP) was used as the detector. For calibrating the diffraction angle, the distance of the IP from the sample (i.e., camera length) was determined by recording the diffraction from silver powder placed on the outer face of the diamond. Average $d$ spacings were obtained by averaging those from the left and the right patterns. This eliminated the error in $d$ spacing arising from that in fixing the center spot on the image plate. Compression run was also repeated using synchrotron radiation from 13B beam line at Photon Factory, KEK, where a 30- $\mu \mathrm{m}$ collimator was used.

Double-sided in situ laser heating was carried out in the diamond-anvil cell using a 100-W cw multimode Nd:YAG laser operating at $1.064 \mu \mathrm{m}$. The laser-heating setup used in the present experiments was similar to that described elsewhere. ${ }^{23}$ The heated area in this system was around

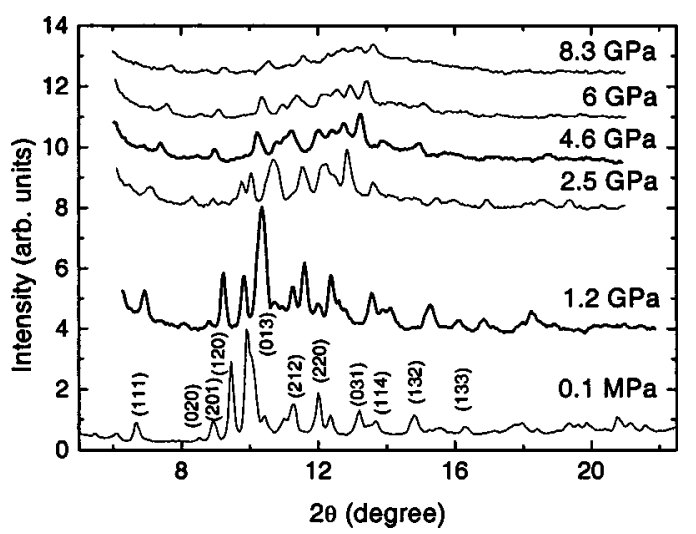

FIG. 1. Diffraction patterns of scandium molybdate at different pressures in the orthorhombic phase.

$25 \mu \mathrm{m}$ in diameter. In order to heat the whole area of the sample within the hole of the gasket, the diamond anvil cell (DAC) was moved in small steps in the $x-y$ plane perpendicular to the direction of the laser beams. On the average, heating at each point was done for about $30 \mathrm{~s}$ and the total heating time was about $30 \mathrm{~min}$. Temperature of the sample during laser heating was estimated by analyzing the spectrum of the black-body radiation from the heated spot using a 300-mm focal length monochromator fitted with CCD detector. For laser-heating runs, no pressure-transmitting medium was used. Pressure was also measured after each heating run. Raman and x-ray diffraction measurements were carried out in the DAC as well as on the recovered samples after releasing the pressure. Microscopic observations were made on polished fragments of the recovered samples after embedding them in an epoxy resin using a JSM-5600 scanning electron microscope.

\section{RESULTS AND DISCUSSIONS}

\section{A. Compression at ambient temperature}

Scandium molybdate belongs to space group Pnab (60) with an orthorhombic unit cell containing four formula units $^{24}$ and cell parameters of $a_{0}=9.6404, b_{0}=9.5481$, and $c_{0}=13.2472 \AA$ at ambient temperature. ${ }^{18}$ All the reported diffraction peaks ${ }^{24}$ were identified in 0.1-MPa patterns, and the cell parameters obtained were in good agreement with those reported earlier. ${ }^{18}$ Figure 1 shows the diffraction patterns at several pressures. At higher pressure, the diffraction peaks exhibited shift to higher $2 \theta$ and changes in their intensities. The intensities of the diffraction peaks decreased rapidly up to $4 \mathrm{GPa}$ and further decrease was much slower. Furthermore, some of the diffraction peaks grew weaker and more rapidly than others. This suggested ${ }^{21}$ a possible evolution of positional disorder of selective atoms at high pressure. Figure 2 shows the pressure dependence of several $d$ spacings. The $d$ spacings decrease monotonically as a function of pressure. The behavior of some of the weak peaks could not be followed up to the highest pressure. The cell parameters of the orthorhombic phase obtained from profile analysis decreased monotonic as a function of pressure to $0.882 a_{0}$, $0.916 b_{0}$, and $0.868 c_{0}$ at $11 \mathrm{GPa}$, indicating that the compression is anisotropic. The unit-cell volume $V$ normalized to the 


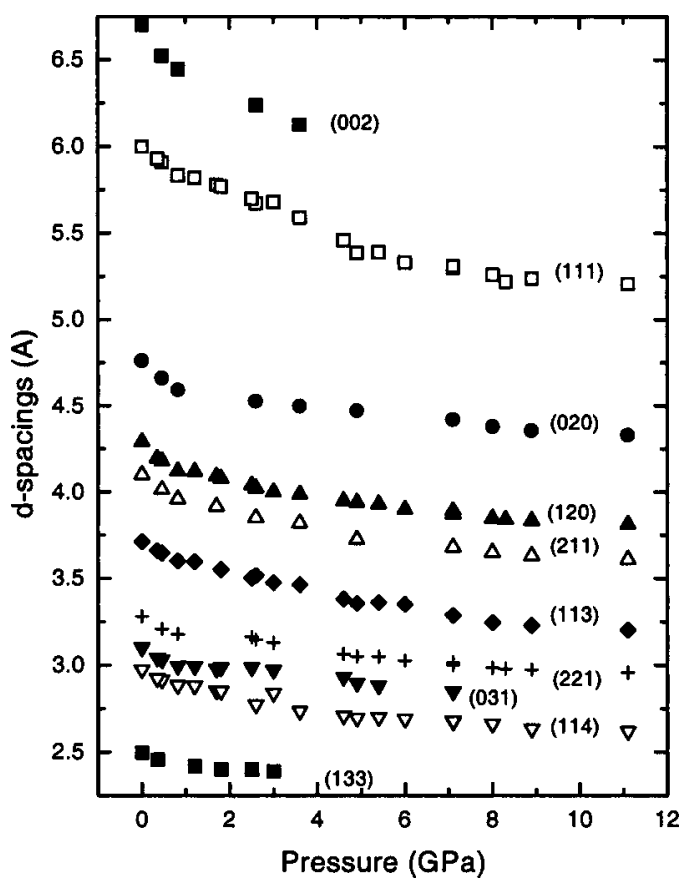

FIG. 2. Pressure dependence of $d$ spacings in scandium molybdate.

ambient pressure volume $V_{0}$ is shown in Fig. 3 as a function of pressure $P$. One can note that at a pressure of $11 \mathrm{GPa}$, the volume reduces to as small as 0.7 of the ambient pressure value, suggesting the compound to be a very soft solid. The $P-V$ data were fitted to the third-order Birch-Murghanan equation of state ${ }^{25}$

$$
P=\frac{3}{2} K_{0}\left(u^{7 / 3}-u^{5 / 3}\right)\left[1-\frac{3}{4}\left(4-K_{0}^{\prime}\right)\left(u^{2 / 3}-1\right)\right],
$$

where $u=V_{0} / V$. The fit yielded the bulk modulus $K_{0}$ as $6 \pm 1 \mathrm{GPa}$ and its pressure derivative $K_{0}^{\prime}$ to be $9 \pm 2$. The fitted equation of state is also shown in Fig. 3. A value of $K_{0}$ $=6 \mathrm{GPa}$ confirms that the compound is highly compressible. Furthermore, a large value of $K_{0}^{\prime}$ implies that as compression proceeds, bulk modulus changes rapidly making further squeezing more difficult. High compressibility of network structures arises because of the presence of considerable amount of empty spaces in between polyhedral structural units. Although several other molybdates have been investigated at high pressure ${ }^{20,26-29}$ from the point of view of amorphization, their equations of state have not been determined.

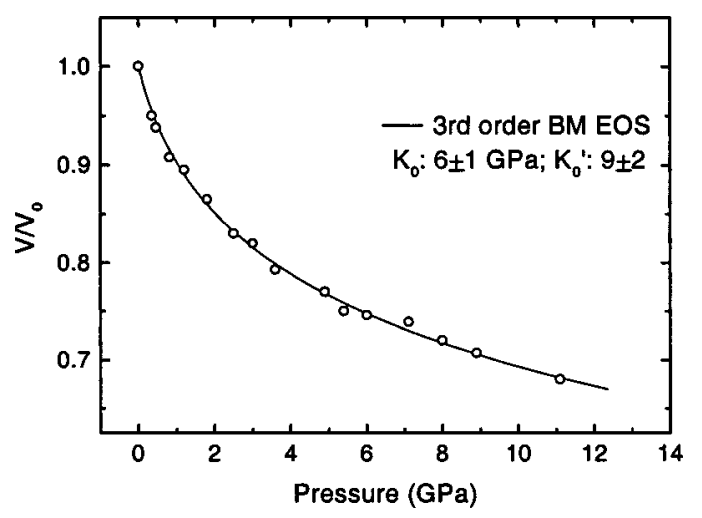

FIG. 3. Relative volume of scandium molybdate as a function of pressure.

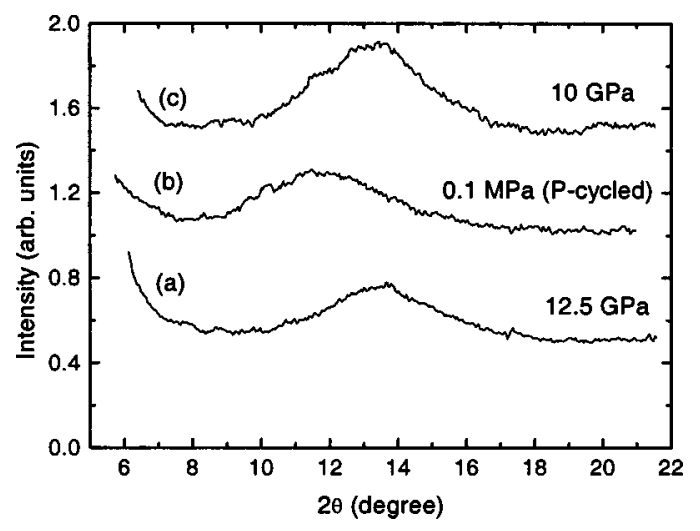

FIG. 4. Diffraction patterns of scandium molybdate (a) at $12.5 \mathrm{GPa}$, (b) at ambient pressure after pressure cycling to $15 \mathrm{GPa}$, and (c) at $10 \mathrm{GPa}$ without pressure-transmitting medium.

It is worth pointing out that the orthorhombic phase of $\mathrm{Sc}_{2}\left(\mathrm{MoO}_{4}\right)_{3}$ transforms to a monoclinic phase upon cooling below $178 \mathrm{~K}$. ${ }^{18}$ This phase with eight formula units per unit cell belongs to the space group $P 2{ }_{1} / a$ and is $1.4 \%$ denser than the orthorhombic phase. Application of pressure is often considered to be equivalent to cooling as both these result in the reduction of volume. In view of this, one may expect that this phase transition could occur at higher temperature under applied pressure. Recently, Paraguassu et al. have argued, ${ }^{26}$ based on the Raman spectra, that this transition occurs at $0.29 \mathrm{GPa}$; however, their energy-dispersive XRD data does not give any evidence for the transition. These authors further claim about the occurrence of another structural transition at $2.7 \mathrm{GPa}$. On the other hand, the present angledispersive XRD measurements clearly show that the diffraction peaks of the orthorhombic phase can be identified and followed up to $11 \mathrm{GPa}$. When pressure is increased beyond $12 \mathrm{GPa}$, all diffraction lines disappeared suggesting amorphization at $12 \mathrm{GPa}$ (Fig. 4). This is in contrast to the conclusion $^{26}$ of the occurrence of amorphization above 3.7 $\mathrm{GPa}$, arrived from the behavior of the Raman modes and energy-dispersive XRD pattern. We have earlier shown that the broadening of the Raman lines arises due to molybdate ion disorder while the crystalline order persists up to $11 \mathrm{GPa}^{21}$

Note the broad amorphouslike background in the pattern at $12.5 \mathrm{GPa}$. The occurrence of PIA in this system, as predicted by the $\Delta V<0$ condition, thus supports the unified model of amorphization/decomposition. ${ }^{15}$ It may be mentioned that if no pressure-transmitting medium is used, amorphization is found to be complete even at 9.5 GPa. Decrease of amorphization pressure under nonhydrostatic conditions due to presence of shear stresses is similar to that found in other compounds. ${ }^{29,30}$ When pressure was reduced to ambient, the broad pattern remained, suggesting irreversibility of amorphization. One can also see from Fig. 4 that the center of the amorphous peak depends on the pressure and shifts to lower $2 \theta$ when pressure is released. This is because of the relaxation of the average nearest-neighbor distance in the amorphous state with only the short-range order.

Figure 5 shows the Raman spectra at several pressures. The Raman spectrum below $450 \mathrm{~cm}^{-1}$ consists of lattice 


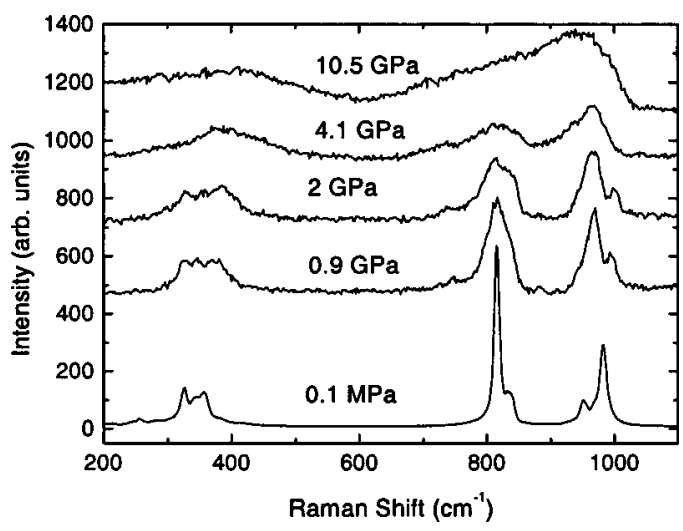

FIG. 5. Raman spectra of scandium molybdate at different pressures.

modes and the $\nu_{2}$ and $\nu_{4}$ bending modes of $\mathrm{MoO}_{4}$ tetrahedra. On the other hand, above $750 \mathrm{~cm}^{-1}$, the modes correspond to only symmetric stretching $\left(\nu_{1}\right)$ and antisymmetric stretching $\left(\nu_{3}\right)$ vibrations. The modes below $200 \mathrm{~cm}^{-1}$ could not be observed using this Raman spectrometer because of attenuation of the intensity due to the use of notch filter for eliminating the Rayleigh background. The nondegenerate symmetric stretching mode $\nu_{1}$ exhibits a set of three peaks at 951,968 , and $982 \mathrm{~cm}^{-1}$, the middle peak being only a shoulder. On the other hand, at $0.9 \mathrm{GPa}$, this shoulder becomes the strongest component and the intensity of the strongest component at $982-\mathrm{cm}^{-1}$ peak reduces drastically. Presence of more peaks than predicted by group theory has been attributed to ${ }^{21}$ the existence of orientational disorder of $\mathrm{MoO}_{4}$ tetrahedra. The change of relative intensities between different components of the same mode as a function of pressure or temperature arises due to the change of orientations of polyatomic ion from one to another. Orientational disorder of polyatomic ions has been shown to cause amorphization. ${ }^{14,31}$ Above $4 \mathrm{GPa}$, the modes become broad rapidly and several weak modes lose their identity and only three broad bands centered at $385\left(\nu_{4}\right), 817\left(\nu_{3}\right)$, and $966\left(\nu_{1}\right) \mathrm{cm}^{-1}$ are seen. The positions of the broad Raman lines were obtained by fitting Lorentzian line shapes with a standard error of about $2 \mathrm{~cm}^{-1}$. The pressure dependencies of the mode frequencies are shown in Fig. 6. Note that the frequencies of several modes increase as a function of pressure, whereas the modes at 815 (the main component of $\nu_{3}$ mode) and $951 \mathrm{~cm}^{-1}$ (low-

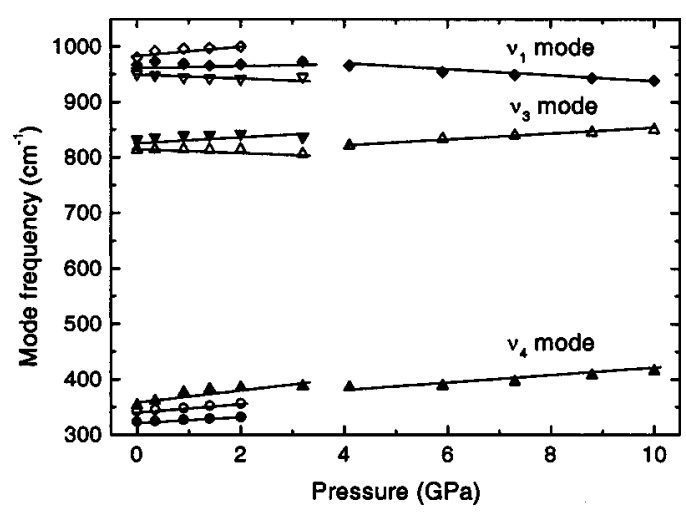

FIG. 6. Pressure dependence of mode frequencies in scandium molybdate. Straight lines through data are guides to the eye. est frequency component of the $\nu_{1}$ triplet) exhibit an opposite behavior. A temperature-dependent Raman spectroscopic study of scandium molybdate has shown ${ }^{32}$ that the phonon frequencies change discontinuously across the orthorhombicmonoclinic transition. The large compressibility of the compound suggests that a volume change of $1.4 \%$ would occur at rather low pressure of only $0.1 \mathrm{GPa}$. Hence if this transition was to occur at high pressure, one should have found the phonon frequencies at the lowest pressure to be different from those at the ambient. Recently, Paraguassu et al. interpreted their Raman results in terms of an orthorhombicmonoclinic transition at $0.29 \mathrm{GPa}$. On the other hand, the present experiments did not show any evidence for the $1000-\mathrm{cm}^{-1}$ peak, characteristic of the monoclinic phase. Furthermore, the changes observed in the relative intensities can be explained on the basis of the evolution of orientational disorder of molybdate ions.

One can see from Fig. 6 that the $\nu_{1}$ mode frequency continues to decrease above $4 \mathrm{GPa}$ also. The decrease of the center frequency of $\nu_{1}$ mode by about $27 \mathrm{~cm}^{-1}$ between 4.1 and $10.5 \mathrm{GPa}$ is clearly evident in the spectrum at $10.5 \mathrm{GPa}$ also (Fig. 5). It may be pointed out that the softening of internal modes of tetrahedral ions is quite uncommon. Only recently, a softening of $\nu_{1}$ mode has been found in the highpressure phase of $\mathrm{Zr}\left(\mathrm{MoO}_{4}\right)_{2}$ prior to its amorphization. ${ }^{27}$ Increase/decrease of the $\nu_{1}$ internal mode frequency of the $X Y_{4}$ tetrahedral molecular ions (such as $\mathrm{SO}_{4}, \mathrm{WO}_{4}$, and $\mathrm{MoO}_{4}$ ) due to the reduction/extension of $X-Y$ bond distance is well established. ${ }^{30,33}$ Hence, the decrease of the $\nu_{1}$ mode frequency during compression observed in the present case implies that $\mathrm{Mo}-\mathrm{O}$ bond distance increases instead of decreasing, suggesting an instability of $\mathrm{MoO}_{4}$ ion at high pressure. The oxygen atoms moving farther away from molybdenum may eventually result in increase of average oxygen coordination around Mo in the disordered state from fourfold to, say, octahedral sixfold. In the related negative thermalexpansion material $\mathrm{Zr}\left(\mathrm{WO}_{4}\right)_{2}$, the instability of tetrahedral oxygen coordination around $\mathrm{W}$ atoms has been conjectured ${ }^{28}$ to be responsible for the breakdown of framework structure and the occurrence of PIA. It may be mentioned that increase of oxygen coordination has also been found in moleculardynamics simulation study of amorphization of quartz. ${ }^{12}$ In addition, broadening of internal modes is also found across amorphization. $^{21,34}$ As mentioned earlier, guided by the changes in the Raman spectra, Paraguassu et al. identified another structural transition at $2.7 \mathrm{GPa}$ prior to amorphization. ${ }^{26}$ The changes in the XRD pattern at $4 \mathrm{GPa}$ were argued to be associated with this transition; however, no attempts were made to identify the structure of the new phase. On the other hand, we have earlier found ${ }^{21}$ that the intensities of some of the diffraction peaks decrease more rapidly than those of others. These anomalous changes in the intensities may make the diffraction pattern appear different and could possibly be misinterpreted as an evidence of a phase transition. The anomalous changes in the intensities have been shown to arise due to the molybdate ion disorder. $^{21}$

Although complete disappearance of diffraction pattern (complete amorphization) occurs at $12 \mathrm{GPa}$, the initial (up to 
$4 \mathrm{GPa}$ ) rapid decrease of diffraction intensities is associated with the molybdate ion disorder in the lattice, ${ }^{21}$ i.e., gross positional and orientational disorder of $\mathrm{MoO}_{4}$ tetrahedra sets in at a much lower pressure than the complete amorphization at $12 \mathrm{GPa}$. In this context, it is important to point out the critical disorder ${ }^{35}$ and critical defect density ${ }^{36}$ models of solid-state amorphization. According to these models, the increase in entropy due to positional disorder lowers the free energy of the amorphous state. Similarly, the orientational disorder of polyatomic ions beyond a critical value has also been shown to cause amorphization. ${ }^{14}$ These are qualitatively analogous to the Lindemann criterion of melting ${ }^{37}$ that states that long-range order is destroyed when atomic displacements are typically $10 \%$ of the interatomic separation. Although the liquid and the amorphous phases differ in terms of their thermodynamic stability and the time scales of atomic diffusion, both of these lack long-range order and hence are often treated as structurally analogous. The central idea of these models, i.e., the existence of a critical disorder required for the loss of long-range order, originally developed for single-component (monatomic) systems can be extended also to compounds, which have different interpenetrating sublattices. The present results on $\mathrm{Sc}_{2}\left(\mathrm{MoO}_{4}\right)_{3}$ suggest that the extent of disorder and its rate of growth in different sublattices could be different, and one sublattice could amorphize (disordered beyond a critical value) earlier than the other did. From this it emerges that amorphization of different sublattices could occur in stages. It may be mentioned that there have been some reports ${ }^{38-41}$ of gradual disorder of $\mathrm{H}$ positions in $\mathrm{Co}(\mathrm{OH})_{2}$. Partial or sublattice amorphization found in this system can be considered as a static analog of the sublattice melting in superionic conductors. The displacements from equilibrium positions in the present case represent frozen-in static disorder, whereas in the ionic conductors it is dynamic in nature.

\section{B. Laser heating of amorphous samples}

In order to examine if a pressure-induced decomposition occurs in this system under HP-HT conditions, pressureamorphized samples were subjected to laser heating inside DAC with different laser powers. In order to exclude the possibility of reaction of the sample with the pressuretransmitting medium at high temperature, no pressuretransmitting medium was used in the heating runs. The sample, which was transparent and colorless at $10 \mathrm{GPa}$ to begin with, was found to turn black immediately upon laser heating. After laser heating the sample x-ray diffraction patterns were recorded at high pressure as well as after releasing the pressure. Pressure was also measured after each heating run and was found to be within $0.5 \mathrm{GPa}$ of that before heating. Figure 7 shows the diffraction patterns at $10 \mathrm{GPa}$ after heating the sample with successively higher laser power. From the spectrum of the incandescent light emitted from the sample, the temperature was estimated to be $1350 \pm 100 \mathrm{~K}$ for heating with $15 \mathrm{~W}$ laser power. No temperature measurement was possible during heating with lower laser powers. One can see that for heating with $8 \mathrm{~W}$, weak and broad diffraction peaks just begin to ride over the amorphous back-

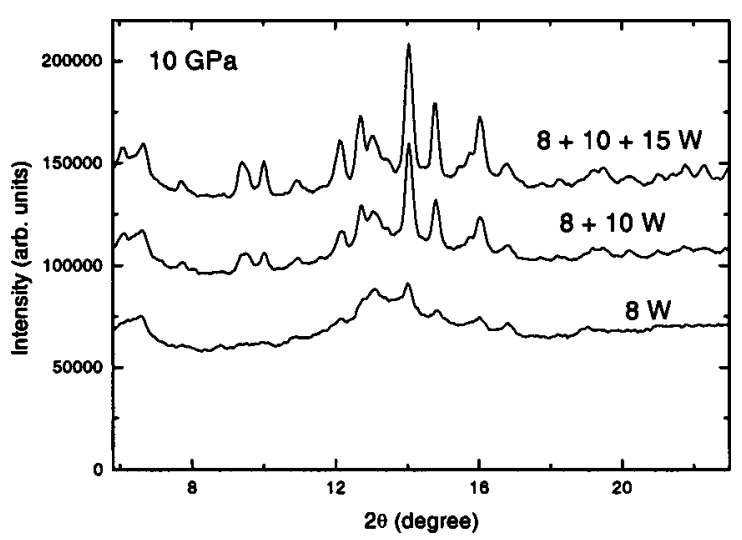

FIG. 7. Diffraction patterns after heating scandium molybdate at $10 \mathrm{GPa}$ inside DAC with different laser powers of YAG laser.

ground. On the other hand, $15 \mathrm{~W}$ power resulted in complete crystallization of the sample. This pattern is different from that of scandium molybdate. When pressure is released, the diffraction pattern does not change. This suggests that either the phase/phases formed as a consequence of laser heating have no structural transition up to $10 \mathrm{GPa}$ or these highpressure phase/phases can be quenched to the ambient.

Figure 8 shows the diffraction pattern of the recovered sample. Here several diffraction peaks are found to agree with those of the orthorhombic phase Pbnm (62) of molybdenum trioxide. This suggests a possible decomposition of the parent compound into $\mathrm{MoO}_{3}$ and other products. In analogy with $\mathrm{Zr}\left(\mathrm{WO}_{4}\right)_{2}$, if one considers this compound also to have undergone decomposition into mixture of simple oxides, the other product could be $\mathrm{Sc}_{2} \mathrm{O}_{3}$. However, it is not necessary that decomposition should always lead to mixture of simple oxides. More complex-mixed oxides have been found as the daughter products ${ }^{42,43}$ in the case $\mathrm{CuGeO}_{3}$ and $\mathrm{Mg}_{2} \mathrm{SiO}_{4}$. In view of this, one needs to examine other mixed oxides of $\mathrm{Sc}_{2} \mathrm{O}_{3}$ and $\mathrm{MoO}_{3}$. The only other known mixed oxide is $\mathrm{Sc}_{2} \mathrm{Mo}_{2} \mathrm{O}_{9} \cdot{ }^{44}$ We find that several of the prominent lines observed in the laser-heated sample correspond to $\mathrm{Sc}_{2} \mathrm{Mo}_{2} \mathrm{O}_{9}$ (Table I). It may be pointed out that although the $d$ spacings for $\mathrm{Sc}_{2} \mathrm{Mo}_{2} \mathrm{O}_{9}$ have been reported, ${ }^{44}$ its pattern is unindexed and structure and cell parameters have not been obtained. In order to know the structure and the unit-cell

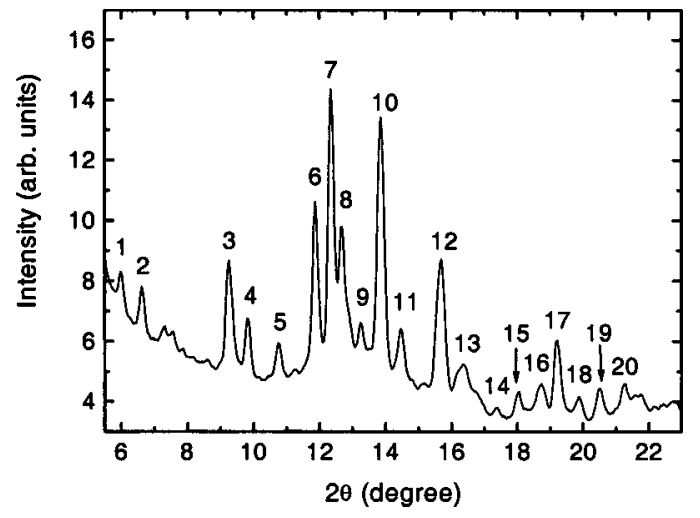

FIG. 8. Diffraction pattern of the sample recovered after laser heating. The peaks labeled 1-20 are indexed to various phases in Table I. The peak labeled 16 could not be indexed. 
TABLE I. Observed $d$ spacings (in $\AA$ ) in the $\mathrm{Sc}_{2} \mathrm{Mo}_{3} \mathrm{O}_{12}$ sample recovered after laser heating at $10 \mathrm{GPa}$. The calculated $d$ values and their indices are also given. Different compounds are labeled as: $\mathrm{S} 12$ : $\mathrm{Sc}_{2} \mathrm{Mo}_{3} \mathrm{O}_{12}, \mathrm{M} 3$ : $\mathrm{MoO}_{3}, \mathrm{~S} 9: \mathrm{Sc}_{2} \mathrm{Mo}_{2} \mathrm{O}_{9}$, and $\mathrm{S} 3: \mathrm{Sc}_{2} \mathrm{O}_{3}$. Some lines have multiple indices.

\begin{tabular}{rccc}
\hline \hline & Observed $d$ spacing & Calculated $d$ spacing & Index \\
\hline 1 & 6.741 & 6.621 & $(002) \mathrm{S} 12$ \\
2 & 6.100 & 6.016 & $(111) \mathrm{S} 12$ \\
3 & 4.378 & 4.521 & $(201) \mathrm{S} 12$ \\
4 & 4.123 & 4.081 & $(211) \mathrm{S} 12$ \\
5 & 3.769 & 3.807 & $(110) \mathrm{M} 3$ \\
6 & 3.413 & 3.440 & $(120) \mathrm{M} 3$ \\
& & 3.377 & $(220) \mathrm{S} 12$ \\
7 & 3.282 & 3.275 & $(121) \mathrm{S} 9$ \\
8 & 3.200 & 3.189 & $(021) \mathrm{M} 3$ \\
9 & 3.061 & 3.075 & $(031) \mathrm{S} 12$ \\
10 & 2.929 & 2.970 & $(420) \mathrm{S} 9$ \\
11 & 2.807 & 2.843 & $(222) \mathrm{S} 3$ \\
12 & 2.591 & 2.612 & $(140) \mathrm{M} 3$ \\
13 & 2.482 & 2.497 & $(041) \mathrm{M} 3$ \\
& & 2.483 & $(133) \mathrm{S} 12$ \\
& & 2.476 & $(240) \mathrm{S} 9$ \\
14 & 2.342 & 2.318 & $(060) \mathrm{M} 3$ \\
15 & 2.256 & 2.276 & $(150) \mathrm{M} 3$ \\
& & 2.257 & $(041) \mathrm{S} 9$ \\
16 & 2.170 & $\cdots$ & $?$ \\
17 & 2.118 & 2.112 & $(141) \mathrm{M} 3$ \\
18 & 2.049 & 2.049 & $(212) \mathrm{S} 9$ \\
19 & 1.986 & 1.979 & $(200) \mathrm{M} 3$ \\
20 & 1.918 & 1.915 & $(720) \mathrm{S} 9$ \\
\hline \hline
\end{tabular}

volume, we carried out a structure search on the reported ${ }^{44}$ pattern and found best fit for an orthorhombic unit cell with $a=14.485(8), b=10.433(5)$, and $c=4.425(2) \AA$ and $Z=4$. For this standard software XPOW supplied with STOE X-ray diffractometer was used. No lines were left unindexed. Although the average $\Delta(2 \theta)$ had a reasonable value of 0.02 , the figure of merit turned out to be only 2.6. This is because the figure of merit is defined as [N(observed)/N(possible)]/ [Average $\Delta(2 \theta)$ ]. The ratio of number of possible lines to those observed in this case was large $(\sim 20)$. It may be pointed out that this data is classified by ICDD as of poor quality "O" due to inadequate range of intensities. Hence, obtaining further structural information from this data was not possible. This fit also allowed us to index the reported pattern. Furthermore, the $100 \%$ (222) peak of $\mathrm{Sc}_{2} \mathrm{O}_{3}$ is also present in the recovered sample as a weak peak. This suggests that a small amount of $\mathrm{Sc}_{2} \mathrm{O}_{3}$ was also formed during laser heating. In addition, a few weak diffraction peaks were found to correspond to the parent compound $\mathrm{Sc}_{2}\left(\mathrm{MoO}_{4}\right)_{3}$. This may be due to recrystallization of the part of the sample that did not experience the highest temperature. Out of the total sample thickness $(70 \mu \mathrm{m})$, the part that is in contact with the diamond faces does not get sufficiently heated due to high thermal conductivity of diamond, while the sample in the interior reaches the maximum temperature. Thus it is likely that the region of the sample in contact with the diamond faces got recrystallized. Thus the present results suggest $\mathrm{MoO}_{3}$ and $\mathrm{Sc}_{2} \mathrm{Mo}_{2} \mathrm{O}_{9}$ to be the main decomposition products, while a small amount of $\mathrm{Sc}_{2} \mathrm{O}_{3}$ is also formed.

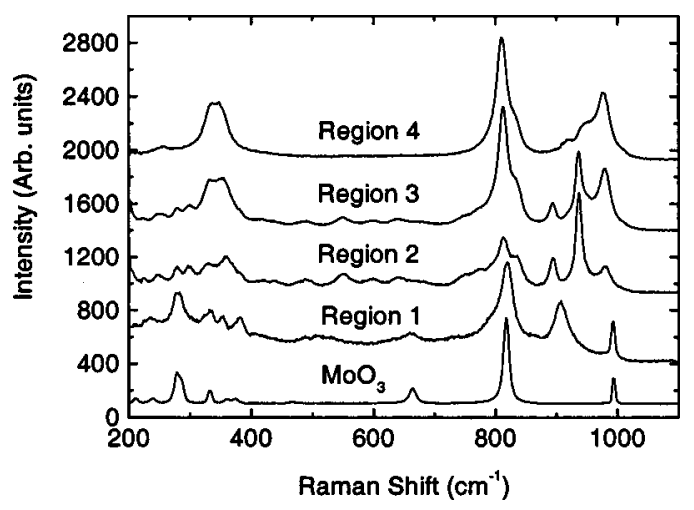

FIG. 9. Raman spectra from different regions of the recovered samples. Spectrum of $\mathrm{MoO}_{3}$ is also shown for comparison.

Some weak peaks could not be indexed, suggesting some additional unidentified phases may also have formed. The $d$ spacings indexed to orthorhombic structures of $\mathrm{MoO}_{3}$, $\mathrm{Sc}_{2} \mathrm{Mo}_{2} \mathrm{O}_{9}$, and $\mathrm{Sc}_{2}\left(\mathrm{MoO}_{4}\right)_{3}$ were used for calculating the cell parameters. For $\mathrm{MoO}_{3}$, these are $a=3.958(5), b$ $=13.91(1)$, and $c=3.589(7) \AA$; for $\mathrm{Sc}_{2} \mathrm{Mo}_{2} \mathrm{O}_{9}, a=14.38(1)$, $b=10.55(1)$, and $c=4.366(5) \AA$; and for $\mathrm{Sc}_{2}\left(\mathrm{MoO}_{4}\right)_{3}, a$ $=9.619(7), b=9.483(9)$, and $c=13.24(1) \AA$. It may be pointed out that the calculated cell parameters are slightly different from those reported in the JCPDS data. However, the calculated cell volumes are close to the reported value. This may be due to a slight departure of the stoichiometry of the daughter phases from their ideal value arising from factors such as dissolution of $\mathrm{Sc}$ in $\mathrm{MoO}_{3}$, etc. This may also be the reason for marginal disagreement between the observed and the calculated $d$ spacings. Stoichiometries obtained from the SEM analysis are discussed later in this section.

As mentioned in the introduction, a large volume reduction of $30.5 \%$ is expected to take place if the decomposition route is that of Eq. (1). It is worth examining whether the dominant decomposition route found in the present studies, i.e.,

$$
\mathrm{Sc}_{2}\left(\mathrm{MoO}_{4}\right)_{3} \rightarrow \mathrm{Sc}_{2} \mathrm{Mo}_{2} \mathrm{O}_{9}+\mathrm{MoO}_{3},
$$

also corresponds to a volume reduction. From the estimated unit-cell volume of 668.6(4) $\AA^{3}$ for $\mathrm{Sc}_{2} \mathrm{Mo}_{2} \mathrm{O}_{9}$, its volume per formula unit turns out to be $167.17 \AA^{3}$. From this we estimate the volume change for this decomposition reaction to be $-28.6 \%$. This is also large and implies that the other decomposition product $\mathrm{Sc}_{2} \mathrm{Mo}_{2} \mathrm{O}_{9}$ is only slightly less dense packed compared to the constituent oxides.

Raman spectra collected from different regions (labeled 1-4) of the recovered sample are compared in Fig. 9 with that of $\mathrm{MoO}_{3}$. One can see that the $815-\mathrm{cm}^{-1}$ peak found in the recovered sample is present in both $\mathrm{MoO}_{3}$ and in the parent compound (Fig. 5). Hence, this peak cannot be used for unambiguous identification of the phases. On the other hand, the sharp peak at $993 \mathrm{~cm}^{-1}$ and also those at 282, 332, and $662 \mathrm{~cm}^{-1}$ provide unambiguous evidence for the presence of $\mathrm{MoO}_{3}$ as one of the decomposition products. Note that in $\mathrm{Sc}_{2}\left(\mathrm{MoO}_{4}\right)_{3}$, the corresponding peak at $982 \mathrm{~cm}^{-1}$ appears as a triplet. Table II gives a list of Raman peaks found in the spectrum of the recovered sample along with those of 
TABLE II. Positions (in $\mathrm{cm}^{-1}$ ) of the peaks in the Raman spectrum of the $\mathrm{Sc}_{2}\left(\mathrm{MoO}_{4}\right)_{3}$ sample recovered after laser heating at $10 \mathrm{GPa}$. The characteristic peaks of $\mathrm{Sc}_{2}\left(\mathrm{MoO}_{4}\right)_{3}, \mathrm{Sc}_{2} \mathrm{O}_{3}$, and $\mathrm{MoO}_{3}$ are also given for comparison. The mode frequencies in bold correspond to the strongest lines.

\begin{tabular}{cccc}
\hline \hline Recovered sample & $\mathrm{MoO}_{3}$ & $\mathrm{Sc}_{2} \mathrm{O}_{3}$ & $\mathrm{Sc}_{2}\left(\mathrm{MoO}_{4}\right)_{3}$ \\
\hline & 211 & 216 & \\
282 & 239 & & \\
325 & & 315 & 326 \\
332 & 332 & & 345 \\
353 & & 355 & 356 \\
385 & & 388 & \\
485 & & $\mathbf{4 1 5}$ & \\
505 & & 492 & \\
662 & & 520 & $\mathbf{8 1 5}$ \\
797 & 662 & & 834 \\
$\mathbf{8 1 8}$ & $\mathbf{8 1 7}$ & & 961 \\
832 & & & 982 \\
934 & & & \\
976 & & & \\
993 & & & \\
\hline
\end{tabular}

$\mathrm{Sc}_{2}\left(\mathrm{MoO}_{4}\right)_{3}, \mathrm{MoO}_{3}$, and $\mathrm{Sc}_{2} \mathrm{O}_{3}$. If one considers spectra collected from different regions of the recovered sample, peaks at $325,353,385,485,505,797,832,916,934$, and $976 \mathrm{~cm}^{-1}$ are found in addition to those of $\mathrm{MoO}_{3}$. Out of these, the ones at $325,353,832$, and $976 \mathrm{~cm}^{-1}$ correspond to $\mathrm{Sc}_{2}\left(\mathrm{MoO}_{4}\right)_{3}$. Other peaks may correspond to the other decomposition product $\mathrm{Sc}_{2} \mathrm{Mo}_{2} \mathrm{O}_{9}$. Its Raman spectrum has not been reported yet. It may be mentioned that although x-ray diffraction showed some evidence for the presence of a small amount of $\mathrm{Sc}_{2} \mathrm{O}_{3}$ in the recovered sample, the strongest Raman peak of $\mathrm{Sc}_{2} \mathrm{O}_{3}$ at $415 \mathrm{~cm}^{-1}$ is not found in the spectrum of the recovered sample. This may be due to insufficient intensity. If the decomposition reaction proceeds for sufficient time, then one could expect sample to become macroscopically inhomogeneous. The extent of inhomogeneous distribution of daughter phases was also examined using the sharp-focus laser beam of the micro-Raman spectrometer. One can see from Fig. 9 that different spectra have different relative intensities of two or more sets of peaks. Region 1

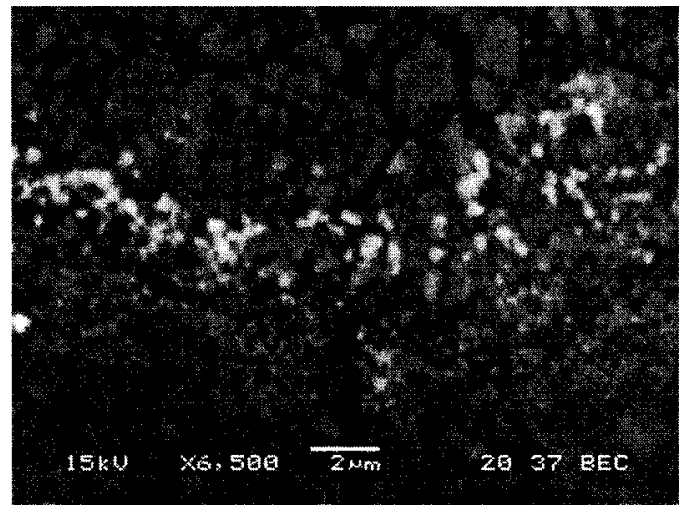

FIG. 10. Scanning electron micrograph (backscattered electron image) of the recovered sample. Brighter regions correspond to the $\mathrm{MoO}_{3}$ phase.

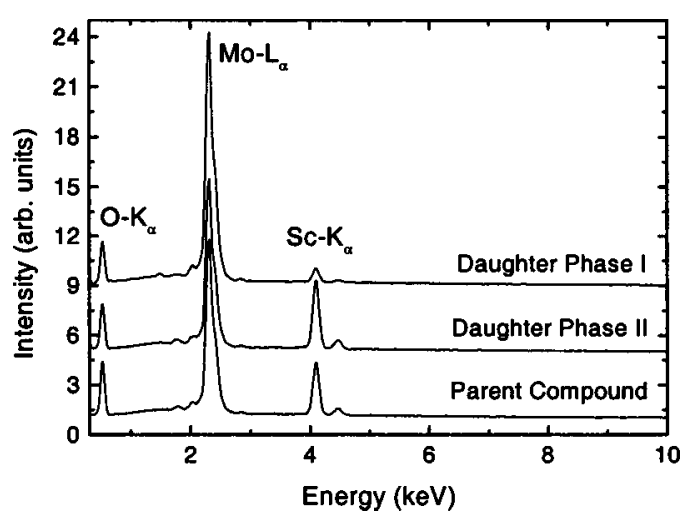

FIG. 11. Energy-dispersive $\mathrm{x}$-ray emission spectra from phase $\mathrm{I}\left(\sim \mathrm{MoO}_{3}\right)$ and phase II $\left(\sim \mathrm{Sc}_{2} \mathrm{Mo}_{2} \mathrm{O}_{9}\right)$ of the sample recovered after laser heating. The spectrum of the original compound scandium molybdate is also shown for comparison.

has the characteristic peaks of $\mathrm{MoO}_{3}$, while region 4 is typical of partially recrystallized $\mathrm{Sc}_{2}\left(\mathrm{MoO}_{4}\right)_{3}$ with no peaks between 400 and $800 \mathrm{~cm}^{-1}$. The regions 2 and 3 have other peaks more dominant than those of $\mathrm{MoO}_{3}$. These results show that the sample becomes inhomogeneous over a micrometer length scale during laser heating resulting in decomposition.

The morphology of the distribution of phases of daughter compounds is also examined using SEM. Figure 10 shows the backscattered electron image of the recovered sample after laser heating. The bright regions, less than a micrometer in size, are rich in Mo. In order to obtain the stoichiometry of these phases, the characteristic x-ray emission spectra from bright (labeled as phase I) and dark (labeled as phase II) regions were analyzed quantitatively. Figure 11 shows the energy-dispersive x-ray emission spectra from regions/phases with different contrast. Note that the intensities of Sc $K_{\alpha}$ line in phases I and II are very different. In the phase I, it is extremely low suggesting that this phase is nearly free from scandium. Also note that in phase II, the Sc $K_{\alpha}$ line is stronger that than that of the parent compound suggesting that this phase is richer in Sc as compared to $\mathrm{Sc}_{2}\left(\mathrm{MoO}_{4}\right)_{3}$. This implies that the parent compound has indeed undergone decomposition into Sc-rich and Sc-depleted phases. Table III gives the composition of two main phases from the x-ray analysis. One can see that the stoichiometry

TABLE III. Composition of phases I and II (bright and dark regions of Fig. 10) obtained from $x$-ray analysis.

\begin{tabular}{lccc}
\hline \hline & $\begin{array}{c}\mathrm{Sc}_{2} \mathrm{O}_{3} \\
(\mathrm{wt} \%)\end{array}$ & $\begin{array}{c}\mathrm{MoO}_{3} \\
(\mathrm{wt} \%)\end{array}$ & \multicolumn{1}{c}{ Stoichiometry } \\
\hline \multicolumn{1}{c}{ Phase I } & & & \\
Spot 1 & 6.1 & 93.9 & $\mathrm{Sc}_{0.13} \mathrm{Mo}_{0.94} \mathrm{O}_{3}$ \\
Spot 2 & 10.6 & 89.4 & $\mathrm{Sc}_{0.22} \mathrm{Mo}_{0.89} \mathrm{O}_{3}$ \\
Spot 3 & 4.4 & 95.6 & $\mathrm{Sc}_{0.09} \mathrm{Mo}_{0.96} \mathrm{O}_{3}$ \\
Ideal value & 0 & 100 & $\mathrm{MoO}_{3}$ \\
\multicolumn{1}{c}{ Phase II } & 33.0 & 67.0 & $\mathrm{Sc}_{2.04} \mathrm{Mo}_{1.98} \mathrm{O}_{3}$ \\
Spot 1 & 34.4 & 65.6 & $\mathrm{Sc}_{2.12} \mathrm{Mo}_{1.94} \mathrm{O}_{3}$ \\
Spot 2 & 30.3 & 69.7 & $\mathrm{Sc}_{1.87} \mathrm{Mo}_{2.06} \mathrm{O}_{3}$ \\
Spot 3 & 32.4 & 67.6 & $\mathrm{Sc}_{2} \mathrm{Mo}_{2} \mathrm{O}_{9}$ \\
Ideal value & & & \\
\hline \hline
\end{tabular}


of phase II deviates slightly from that of $\mathrm{Sc}_{2} \mathrm{Mo}_{2} \mathrm{O}_{9}$ and there is some amount of $\mathrm{Sc}_{2} \mathrm{O}_{3}$ present (dissolved) in $\mathrm{MoO}_{3}$ (phase I) as impurity. These results confirm that the daughter phases have stoichiometry close to those of $\mathrm{MoO}_{3}$ and $\mathrm{Sc}_{2} \mathrm{Mo}_{2} \mathrm{O}_{9}$. As suggested earlier, one can attribute the difference between the observed and calculated cell parameters to the departure from the ideal stoichiometry.

\section{SUMMARY AND CONCLUSIONS}

In situ X-ray diffraction and Raman spectroscopic studies are carried out on negative thermal-expansion material $\mathrm{Sc}_{2}\left(\mathrm{MoO}_{4}\right)_{3}$ at high pressure and high temperature in a YAG laser-heated diamond-anvil cell. The compound has a rather low bulk modulus of $\sim 6 \mathrm{GPa}$. The molybdate ion disorder occurs at a much lower pressure than the amorphization at $12 \mathrm{GPa}$. Furthermore, the softening of the internal modes of molybdate ion suggests its instability and a possible coordination change. As predicted by a recent model of amorphization/decomposition, this compound exhibits PIA at ambient temperature and PID into dense-packed daughter phases at $10 \mathrm{GPa}$ and $1350 \mathrm{~K}$. The daughter phases are identified as $\mathrm{MoO}_{3}$ and $\mathrm{Sc}_{2} \mathrm{Mo}_{2} \mathrm{O}_{9}$.

\section{ACKNOWLEDGMENTS}

It is a pleasure to thank V. S. Sastry and Dr. R. Nithya for fruitful discussions and F. Sakai for help in SEM that was carried out using the facilities of the Materials Design and Characterization Laboratory, ISSP, University of Tokyo. One of the authors (A.K.A.) acknowledges JSPS for travel support and local hospitality at ISSP, and Photon Factory, KEK for the beam-time and also Dr. B. Viswanathan for interest in the work and Dr. Baldev Raj for support and encouragement.

${ }^{1}$ A. K. Arora, in High Pressure Phenomena (International School of Physics "Enrico Fermi" Vol. 147), edited by R. J. Hemley, G. L. Chiarotti, M. Bernasconi, and L. Ulivi, (IOS, Amsterdam, 2002), p. 545, and references therein.

${ }^{2}$ P. Richet and P. Gillet, Eur. J. Mineral. 9, 907 (1997).

${ }^{3}$ R. J. Hemley, A. P. Jephcoat, H. K. Mao, L. C. Ming, and M. H. Manghanani, Nature (London) 334, 52 (1988).

${ }^{4}$ A. K. Arora and T. Sakuntala, J. Phys.: Condens. Matter 4, 8697 (1992).

${ }^{5}$ K. Fuchizaki, S. Sugiyama, and Y. Fujii, J. Chem. Phys. 112, 10379 (2000).

${ }^{6}$ O. Mishima, L. D. Calvert, and E. Whalley, Nature (London) 310, 393 (1984).

${ }^{7}$ G. Richard and P. Richet, J. Geophys. Res. 95, 21549 (1990).

${ }^{8}$ T. R. Ravindran, A. K. Arora, and T. A. Mary, Phys. Rev. Lett. 84, 3879 (2000).

${ }^{9}$ A. Jayaraman, S. Y. Wang, and S. K. Sharma, Solid State Commun. 93, 885 (1995)

${ }^{10}$ I. Daniel, P. Gillet, P. F. McMillan, G. F. Wolf, and M. Verhelst, J. Geo- phys. Res. 102, 10313 (1997)

${ }^{11}$ O. Mishima, L. D. Calvert, and E. Whalley, Nature (London) 314, 76 (1985).

${ }^{12}$ J. S. Tse and D. D. Klug, Phys. Rev. Lett. 67, 3559 (1991).

${ }^{13}$ R. R. Winters, G. C. Serghiou, and W. S. Hammack, Phys. Rev. B 46, 2792 (1992).

${ }^{14}$ T. Sakuntala, A. K. Arora, N. V. C. Shekar, and P. C. Sahu, Europhys. Lett. 44, 728 (1998).

${ }^{15}$ A. K. Arora, Solid State Commun. 115, 665 (2000).

${ }^{16}$ T. R. Ravindran, A. K. Arora, and T. A. Mary, J. Phys.: Condens. Matter 13, 11573 (2001).

${ }^{17}$ J. M. G. Amores, U. Amador, E. Moran, and M. A. A. Franco, Int. J. Inorg. Mater. 2, 123 (2000).

${ }^{18}$ J. S. O. Evans and T. A. Mary, Int. J. Inorg. Mater. 2, 143 (2000).

${ }^{19}$ R. A. Secco, H. Liu, N. Imanaka, G. Adachi, and M. D. Rutter, J. Phys. Chem. Solids 63, 425 (2002).

${ }^{20}$ V. Dimitriev, V. Sinitsyn, R. Dilanian, D. Machon, A. Kuznetsov, E. Ponyatovsky, G. Lucazeau, and H. P. Weber, J. Phys. Chem. Solids 64, 307 (2003).

${ }^{21}$ A. K. Arora, R. Nithya, T. Yagi, N. Miyajima, and T. A. Mary, Solid State Commun. 129, 9 (2004).

${ }^{22}$ A. K. Arora, V. S. Sastry, P. Ch. Sahu, and T. A. Mary, J. Phys.: Condens. Matter 16, 1025 (2004).

${ }^{23}$ T. Yagi, T. Kondo, T. Watanuki, O. Shimomura, and T. Kikegawa, Rev. Sci. Instrum. 72, 1293 (2001).

${ }^{24}$ Powder Diffraction File (JCPDS International Center for Diffraction Data), Card No. 18-1157 (Swarthmore, 1982)

${ }^{25}$ J. Li, H. K. Mao, Y. Fei, E. Gregoryanz, M. Eremets, and C. S. Zha, Phys. Chem. Miner. 29, 166 (2002).

${ }^{26}$ W. Paraguassu et al., Phys. Rev. B 69, 094111 (2004).

${ }^{27}$ D. V. S. Muthu, B. Chen, J. M. Wrobel, A. M. K. Andersen, S. Carlson, and M. B. Kruger, Phys. Rev. B 65, 064101 (2002).

${ }^{28}$ A. Grzechnik and W. A. Crichton, Solid State Sci. 4, 1137 (2002).

${ }^{29}$ A. Jayaraman, S. K. Sharma, Z. Wang, S. Y. Wang, L. C. Ming, and M. H. Manghanani, J. Phys. Chem. Solids 54, 827 (1993).

${ }^{30}$ A. K. Arora, in Adv. in High Pressure Science and Technology, edited by M. Yousuf, N. Subramanian, and K. G. Rajan (Universities Press, Hyderabad, 1997), p. 162

${ }^{31}$ T. Sakuntala, A. K. Arora, N. V. C. Shekar, and P. C. Sahu, J. Phys.: Condens. Matter 12, 4417 (2000).

${ }^{32}$ T. R. Ravindran and A. K. Arora, preprint \#183557 (2004).

${ }^{33}$ A. K. Arora, in Proceedings of the 15th International Conference on Raman Spectroscopy, Pittsburgh, Aug. 11-16, 1996, edited by S. A. Asher and P. B. Stein (Wiley, Chichester, 1996), p. 1056.

${ }^{34}$ M. B. Kruger, Q. Williams, and R. Jeanloz, J. Chem. Phys. 91, 5910 (1989).

${ }^{35}$ J. P. Riviere, Radiat. Eff. 33, 21 (1974).

${ }^{36}$ H. J. Fecht, Nature (London) 356, 133 (1992).

${ }^{37}$ F. A. Lindemann, Phys. Z. 11, 609 (1910).

${ }^{38}$ J. H. Nguyen, M. B. Kruger, and R. Jeanloz, Phys. Rev. B 49, 3734 (1994).

${ }^{39}$ J. H. Nguyen, M. B. Kruger, and R. Jeanloz, Phys. Rev. Lett. 78, 1936 (1997).

${ }^{40}$ J. B. Parise, J. S. Loveday, R. J. Nelmes, and H. Kagi, Phys. Rev. Lett. 83, 328 (1999).

${ }^{41}$ S. R. Shieh and T. S. Duffy, Phys. Rev. B 66, 134301 (2002).

${ }^{42}$ W. Hegenbart, F. Rau, and K. J. Range, Mater. Res. Bull. 16, 413 (1981).

${ }^{43}$ T. Kato et al., Rev. High Pressure Sci. Technol. 7, 119 (1998).

${ }^{44}$ Powder Diffraction File (JCPDS International Center for Diffraction Data Card No. 30-1118 (Swarthmore, 1982). 